\title{
Empowering trainees to raise concerns: a formalised structure for reporting unsafe staffing levels in a university teaching hospital
}

\author{
Author: Aarij S Siddiqui
}

\begin{abstract}
Aims
To create a protocol for trainees to follow when staffing shortages occur which have the potential to compromise patient safety.

\section{Methods}

Frequent on-call rota gaps had left trainees working with understaffed teams. Trainees raised concerns to the Royal College of Physicians (RCP) associate college tutors and the RCP chief registrar that they were not aware how to formally raise concerns regarding unsafe staffing levels, and were uncertain how to escalate concerns out of hours.

We investigated the organisation of the overnight medical teams and researched any existing protocols regarding raising staffing concerns. We also analysed data from the 'Hospital at night' system regarding rota gaps, and undertook a survey of junior doctors' experience while on call.
\end{abstract}

\section{Results}

Twenty junior doctors attended a forum regarding safety concerns associated with current staffing levels. We found that there was no formal protocol for trainees to follow when working understaffed shifts. The actions taken by the trainees were very variable.

Of the 20 junior doctors surveyed, $95 \%$ had worked an on-call shift over the last 3 months with at least one doctor short; $60 \%$ felt stressed about working in understaffed rosters and $70 \%$ felt that patient safety was at risk of being compromised due to staffing shortage. Fortunately, $100 \%$ felt they would be able to phone the consultant on-call regarding any concerns during the shift.

\section{Conclusion}

The RCP chief registrar for the health board relayed the trainees' concerns to multiple senior medical figures within the health board, including the executive medical director and the clinical director for medicine. The areas requiring immediate attention were

Author: Royal College of Physicians chief registrar, Cardiff and Vale University Health Board, Wales, UK highlighted, including the development of an agreed protocol for trainees to follow out of hours when staff shortages may affect patient safety.

The following 'four-step plan' has been agreed for trainees to follow.

1. Inform the consultant on call and clearly state their concerns.

2. Inform the site manager of the staff shortage, who can authorise locum cover and move trainees between specialties overnight if required.

3. At the end of the shift or shifts, fill out a 'Datix' or incident form.

4. Record and reflect on the shift (as per General Medical Council guidance), either through an ePortfolio reflection or a selfaddressed email.

Trainees have been made aware of this four-step plan through the junior doctors' forum and via email. Posters highlighting this stepwise plan and how to fill in a 'Datix' or incident form were distributed during spring 2018.

\section{Conflict of interest statement}

None declared. 